\title{
Prevalence and molecular subtyping of Blastocystis in patients with Clostridium difficile infection, Singapore
}

Lei Deng ${ }^{1,2}$, Huiyi Tay ${ }^{1}$, Guangneng Peng ${ }^{2}$, Jonathan W. J. Lee ${ }^{3,4}$ and Kevin S. W. Tan ${ }^{\text {* }}$

\begin{abstract}
Background: Blastocystis is a common anaerobic colonic protist in humans with controversial pathogenicity. Clostridium difficile (C. difficile) is the commonest cause of infectious diarrhea in healthcare settings. The prevalence and subtype (ST) characteristics of Blastocystis in patients with C. difficile infection (CDI) are rarely documented. Therefore, the present study was conducted to investigate the prevalence and subtype characteristics of Blastocystis in patients with suspicion of CDI in Singapore.

Methods: Fecal samples were collected from 248 patients presenting with suspected CDI from a single tertiary hospital in Singapore. C. difficile was diagnosed through positive glutamate dehydrogenase (GDH) with or without toxin A/B using enzyme immunoassay methods. The prevalence and subtype genetic characteristics of Blastocystis were determined by polymerase chain reaction (PCR) amplification and analysis of the barcode region of the SSU rRNA gene.
\end{abstract}

Results: The proportion of C. difficile in patients with healthcare-associated diarrhea in this study was 44\% (109/248). Among the 109 C. difficile-positive patients, 59 (54.1\%, 59/109) tested positive for toxigenic C. difficile, which was considered CDI. Based on the sequence analyses of the barcode region of the SSU rRNA gene, $10.1 \%(25 / 248)$ of the patients were found to be Blastocystis-positive, and three subtypes were identified: ST7 (64\%, 16/25), ST1 (20\%, 5/25), and ST3 $(16 \%, 4 / 25)$. Remarkably, we found five patients with Blastocystis and C. difficile coinfection, and further subtype analysis showed two with ST7, two with ST1, and one with ST3.

Conclusions: To the best of our knowledge, this is the first study to investigate the subtype distributions of Blastocystis in patients with CDI in Singapore. We found ST7 to be the predominant subtype in diarrheal patients. The pathogenicity of ST7 has been strongly suggested in previous in vitro and mouse model experiments, further confirming its potential pathogenicity to humans.

Keywords: Blastocystis, Clostridium difficile, Pathogenicity, Diarrhea, ST7

*Correspondence: mictank@nus.edu.sg

${ }^{1}$ Laboratory of Molecular and Cellular Parasitology, Healthy Longevity Translational Research Programme and Department of Microbiology and Immunology, Yong Loo Lin School of Medicine, National University of Singapore, Singapore 117545, Singapore

Full list of author information is available at the end of the article

\section{Background}

Blastocystis, an anaerobic colonic protistan parasite, can colonize the intestines of humans and a wide range of animals [1]. Although Blastocystis has been known for more than 100 years, its pathogenicity is still a matter of debate [2]. To date, 22 subtypes have been identified based on analyses of the small subunit $(S S U)$ rRNA gene, while ST21 and ST23-26 still need further data to determine [3]. ST1-9 and ST12 were identified in humans, 
with ST1-4 the most prevalent [4]. A more recent study revealed that ST10 and ST14 are also able to infect humans [5]. The extensive variations in virulence factors and genetic characteristics among Blastocystis subtypes have been identified in vitro and in genomic studies $[6$, 7].

Blastocystis has been previously reported to play a central role in modulating the gut microbiota, whereby Blastocystis colonization can increase the bacterial diversity and abundance of Clostridia [8-10], known producers of short-chain fatty acids, which are usually associated with a healthy gut microbiota [11]. However, other microbiome studies showed that colonization of Blastocystis reduced the proportion of beneficial bacteria, such as Bifidobacterium, in a mouse model and in patients with irritable bowel syndrome (IBS) $[12,13]$. The discrepancy in these results may be due to the enormous genetic variations between different subtypes of Blastocystis, which could result in differences in pathogenic potential.

Clostridium difficile ( $C$. difficile), an important nosocomial pathogen, is the most common causative agent of antibiotic-associated diarrhea [14]. The use of antibiotics in patients with diarrhea can increase the risk of $C$. difficile infection (CDI) by causing dysbiosis [15]. It has been reported that $\mathrm{CDI}$ is one of the most common healthcare-associated infections (HAIs) in the United States [16], and CDI can increase in-hospital mortality, prolong hospitalization, and increase medical costs [17]. Pathogenic $C$. difficile strains are armed with multiple toxins, of which the major toxins are TcdA (also known as ToxA) and TcdB (also known as ToxB) [18]. These toxins disrupt the epithelial cell cytoskeleton and cause the disassociation of tight junctions between colonic epithelial cells and loss of epithelial integrity, leading to diarrhea and inflammation in infected patients $[19,20]$.

To date, there has been only one study reporting the coinfection of Blastocystis and C. difficile in diarrheal patients from the community and healthcare facilities in Colombia [21]. However, the prevalence of Blastocystis and C. difficile in diarrheal patients in Singapore is less reported, and the genetic characteristics of different subtypes of Blastocystis in CDI patients has yet to be reported. Therefore, this study aims to determine the prevalence of Blastocystis and C. difficile in patients from a healthcare facility in Singapore, and to further investigate the subtype distributions and genetic characteristics of Blastocystis among patients with CDI.

\section{Methods}

\section{Sample collection}

A total of 248 consecutive stool samples from unique individuals were collected from the National University Hospital (NUH) (Additional file 1: Table S1), a tertiary care hospital of 1200 beds, and a major referral center with over 50 medical, surgical, and dental specialties. Sampling was conducted between 2017 and 2019. Samples were only from patients with suspected CDI, defined as having diarrhea arising $>72 \mathrm{~h}$ after hospital admission, either receiving or with recent previous antibiotics use, whereby diarrhea was defined as three or more loose stools per day, and defined as type 5 and above on the Bristol stool chart [22]. The study design and protocol were approved by the Domain Specific Review Board of the National Healthcare Group.

\section{Detection and toxigenic profile of $C$. difficile}

The stool samples were first screened for antigens and toxins using the C. DIFF QUIK CHEK COMPLETE ${ }^{\circledR}$ kit (D-EIA; TechLab, Blacksburg, VA) according to the manufacturer's instructions [23]. Briefly, $25 \mu \mathrm{L}$ of stool specimens was added in a tube containing $750 \mu \mathrm{L}$ diluent and one drop of conjugate (TechLab). The specimen was mixed either by vortexing or by repeatedly inverting the tube, and then transferred to the device sample well. After incubation for $15 \mathrm{~min}$ at room temperature, the wash buffer and the substrate (TechLab) were added to the reaction window. Results were read 10 min later. Glutamate dehydrogenase (GDH) antigen and/or toxins were reported positive if a visible band was seen on the antigen and/or the toxin side of the device display window, respectively. All of the above clinical investigations were conducted by NUH's Department of Laboratory Medicine, which is accredited by the Singapore Accreditation Council.

\section{DNA extraction and PCR amplification}

Genomic DNA was extracted from stool samples and Blastocystis ST7 pure cultures (positive control) using the Qiagen DNA Stool Mini Kit (Qiagen, Hilden, Germany) according to the manufacturer's instructions. All samples were screened for the presence of Blastocystis by polymerase chain reaction (PCR) amplification of the barcode region (a fragment of $\sim 600 \mathrm{bp}$ ) of the $S S U$ rRNA gene using the primers BhRDr (5'-GAG CTT TTT AAC TGC AAC AAC G-3') and RD5 (5'-ATC TGG TTG ATC CTG CCA GTA-3') [24]. Reagents per $25 \mu$ reaction were as follows: $12.5 \mu$ GoTaq $^{\circledR}$ DNA polymerase containing $\mathrm{MgCl}_{2}$ (Promega, WI, USA), $1 \mu$ forward primer $(0.4 \mu \mathrm{M}), 1 \mu \mathrm{l}$ reverse primer $(0.4 \mu \mathrm{M}), 2 \mu \mathrm{l}$ genomic DNA, and nuclease-free water to the desired volume. The PCR was started at $94{ }^{\circ} \mathrm{C}$ for $4 \mathrm{~min}$ followed by 30 cycles of $95^{\circ} \mathrm{C}$ for $15 \mathrm{~s}, 60^{\circ} \mathrm{C}$ for $15 \mathrm{~s}$, and $72{ }^{\circ} \mathrm{C}$ for $30 \mathrm{~s}$, with an extension at $72{ }^{\circ} \mathrm{C}$ for $5 \mathrm{~min}$. Positive DNA (ST7) and negative control (nuclease-free water) was included in all of the PCR tests. The PCR products were subjected to electrophoresis in $1.5 \%$ agarose gel (Life Technologies 
Corporation, CA, USA) stained with SYBR Safe (Life Technologies).

\section{Detection and subtyping of Blastocystis}

PCR products with expected fragments (around $600 \mathrm{bp}$ ) were subsequently cleaned up using the QIAquick ${ }^{\circledR}$ PCR Purification Kit according to the manufacturer's instructions (Qiagen, Hilden, Germany) and sent for sequencing (Axil Scientific Pte Ltd, Singapore). Raw sequencing data were checked using Chromas 2.6.6 software (Technelysium, Brisbane, Australia) to guarantee the accuracy of nucleotides. The ambiguous bases at the starts and ends of the sequences were trimmed. The clean sequences then were subjected to BLAST searches (http://www. ncbi.nlm.nih.gov/blast/), and the reference sequences were downloaded from the GenBank database. Blastocystis subtypes were identified by BLAST searches (http:// blast.ncbi.nlm.nih.gov/Blast.cgi) and the alleles were identified at the Blastocystis database (http://pubmlst. org/blastocystis).

\section{Phylogenetic analysis}

A dataset was assembled including the clean sequences obtained in the present study and reference sequences encompassing ST1-17 except for ST11, and an alignment was carried out using the MUSCLE algorithm of MEGA X [25]. The alignment was trimmed using MEGA 6 (http://www.megasoftware.net/), with about a 600-bp barcode region remaining. The barcode region of ST11 is not available, so we excluded ST11 in the phylogenetic analysis [3]. ST21 and ST23-26 were not included because these subtypes require further data for confirmation. The phylogenetic tree was constructed with the neighbor-joining (NJ) method using MEGA 6 software. Evolutionary distances were calculated using the Kimura two-parameter model. The reliability of the trees was assessed by bootstrap analysis with 1000 replicates.

\section{Statistical analysis}

Statistical analyses were performed using SPSS version 22.0 (IBM Corp., Armonk, NY, USA). A chi-square $\left(x^{2}\right)$ test and 95\% confidence intervals (CIs) were used to compare the prevalence of Blastocystis between different groups. Differences were considered statistically significant at $P$-values $<0.05$. Odds ratios (ORs) were also calculated to explore the strengths of association between Blastocystis positivity and gender, age, and ethnicity.

\section{Results}

\section{Prevalence of $C$. difficile in patients with diarrhea}

Among the 248 diarrheal stool samples, we found that 139 (56\%) samples were negative for $C$. difficile (group A), while 109 (44\%) specimens were positive for the C. difficile GDH antigen. Furthermore, in terms of the 109 C. difficile-positive specimens, 50 (45.9\%) of these patients had non-toxigenic $C$. difficile (group B), and 59 (54.1\%) tested positive for toxigenic C. difficile (group C). It should be noted that CDI was considered only when both the GDH antigen and toxin were positive.

\section{Prevalence of Blastocystis in patients with diarrhea}

It is worth noting that since normal PCR was used instead of qPCR, the Blastocystis prevalence here refers to the minimum prevalence. In total, Blastocystis was detected in 25 of 248 fecal samples (10.1\%). Specifically, of the 25 Blastocystis-positive patients, 13 were found in group A (9.4\%), and seven (14\%) and five (8.5\%) were identified in group $B$ and group $C$, respectively (Table 1 ). The difference in Blastocystis prevalence was nonsignificant among different groups $(P>0.05)$.

The prevalence of Blastocystis in male and female patients was $13.5 \%$ and $7.6 \%$, respectively (Table 2), and the difference was not significant $(P>0.05)$. Across age groups, the highest prevalence of Blastocystis was observed in ages 18-64 years $(13 \%, 14 / 108)$, followed by $\geq 65$ years $(8.8 \%, 11 / 125)$. Blastocystis was not detected in young patients (age $<17$ years). Similarly, there was no significant difference in the prevalence of Blastocystis among different age groups $(P>0.05)$ (Table 2). The prevalence of Blastocystis among various ethnic groups is also presented in Table 2, with the highest prevalence being observed in Indian populations $(17.2 \%, 5 / 29)$ and the lowest prevalence in Malay patients $(2.6 \%, 1 / 39)$; however, the difference was not significant

Table 1 The prevalence and subtype distributions of Blastocystis among different groups

\begin{tabular}{|c|c|c|c|c|c|c|}
\hline Groups & No. examined & No. positive & Prevalence (\%) (95\%) & OR (95\%) & $P$ value & Subtypes (n) \\
\hline Group A & 139 & 13 & $9.4 \%(4.5-14.2)$ & Reference & & ST7 (9), ST3 (3), ST1 (1) \\
\hline Group B & 50 & 7 & $14 \%(4.4-23.6)$ & $1.615(0.605-4.3110)$ & 0.363 & ST7 (5), ST1 (2) \\
\hline Group C & 59 & 5 & $8.5 \%(1.4-15.6)$ & $0.919(0.312-2.703)$ & 0.844 & ST7 (2), ST1 (2), ST3 (1) \\
\hline Total & 248 & 25 & $10.1 \%$ & & & ST7 (16), ST1 (5), ST3 (4) \\
\hline
\end{tabular}

Group A represents C. difficile antigen-negative patients; group B represents $C$. difficile antigen-positive and toxigenic-negative patients; group $C$ represents both $C$. difficile antigen- and toxigenic-positive patients 
Table 2 The prevalence of Blastocystis in diarrheal patients by gender, age, and ethnicity

\begin{tabular}{|c|c|c|c|c|c|}
\hline Characteristics & No. of examined & No. of positive & Prevalence (\%) (95\%) & OR (95\%) & $P$ value \\
\hline \multicolumn{6}{|l|}{ Gender } \\
\hline Male & 104 & 14 & $13.5 \%(6.9-20)$ & $1.881(0.817-4.33)$ & 0.138 \\
\hline Female & 144 & 11 & $7.6 \%(3.3-12)$ & Reference & \\
\hline \multicolumn{6}{|l|}{ Age (years) } \\
\hline$\leq 17$ & 15 & 0 & 0 & 0 & 0.999 \\
\hline $18-64$ & 108 & 14 & $13 \%(6.6-19.3)$ & $1.554(0.669-3.56)$ & 0.309 \\
\hline$\geq 65$ & 125 & 11 & $8.8 \%(3.8-13.8)$ & Reference & \\
\hline \multicolumn{6}{|l|}{ Ethnicity } \\
\hline Chinese & 150 & 16 & $10.7 \%(5.7-15.6)$ & $1.075(0.293-3.945)$ & 0.914 \\
\hline Malay & 39 & 1 & $2.6 \%(2.4-7.5)$ & $0.237(0.023-2.401)$ & 0.223 \\
\hline Indian & 29 & 5 & $17.2 \%(3.5-31.0)$ & $1.875(0.405-8.688)$ & 0.422 \\
\hline Others & 30 & 3 & $10 \%(0.7-20.1)$ & Reference & \\
\hline \multicolumn{6}{|l|}{ Antibiotics } \\
\hline No indicated & 226 & 24 & $10.6 \%(6.6-14.6)$ & $2.495(0.321-19.386)$ & 0.382 \\
\hline Treated & 22 & 1 & $4.5 \%(4.2-13.2)$ & Reference & \\
\hline Total & 248 & 25 & $10.1 \%$ & & \\
\hline
\end{tabular}

$(P>0.05)$. The prevalence of Blastocystis in antibiotictreated and untreated patients was $4.5 \%$ and $10.6 \%$, respectively, and this difference was also not significant $(P>0.05)$ (Table 2).

\section{Subtype distributions of Blastocystis in patients with diarrhea}

A total of three subtypes (ST1, ST3, and ST7) were identified based on analysis of the barcode region of the SSU rRNA gene (Table 1). ST7 was the most common subtype $(64 \%, 16 / 25)$, followed by ST1 $(20 \%, 5 / 25)$ and ST3 (16\%, 4/25). Based on the analysis of Blastocystis multilocus sequence typing, for the ST7 isolates four alleles were identified $(99,100,101$, and 137), for ST1 only one allele (4), and for ST3 also one allele (36). Remarkably, we found five patients with Blastocystis and C. difficile coinfection (Table 1). Subtype analysis revealed two with ST7, two with ST1, and one with ST3, and there was no significant difference in prevalence between different subtypes $(P>0.05)$ (Fig. 1). In contrast, $13 C$. difficile-negative patients were positive for Blastocystis infection, and the prevalence of ST7 $(6.5 \%, 9 / 139)$ was significantly higher than ST3 $(2.2 \%, 3 / 139)$ and ST1 $(0.7 \%, 1 / 139)(P<0.05)$ (Fig. 1).

\section{Genetic characteristics of Blastocystis subtypes}

A total of 12 representative sequences were obtained from the 25 Blastocystis-positive samples in the present study, including one ST1 sequence, two ST3 sequences, and nine ST7 sequences. The sequence of ST1/allele 4 (MT974098) derived from strains C34, C41, C54, and A37 was identical to that of a Blastocystis sample found in sewage water in the Philippines (KY964542). Similarly, the two sequences of ST3/allele 36 (MT974099

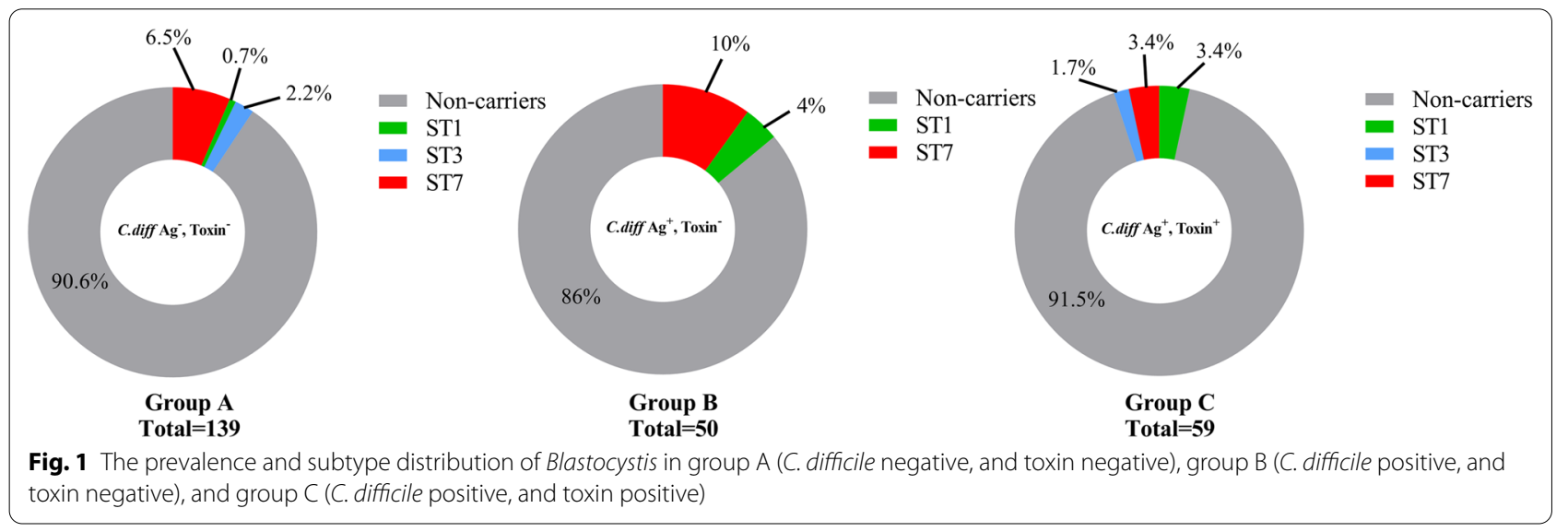


and MT974010) obtained from strains C6 and N38 showed $100 \%$ identity to that of Blastocystis reported from humans in Japan (KT438691 and KT438689, respectively).

ST7 showed high genetic variations within the 600bp barcode region of SSU rRNA of Blastocystis. Among the nine ST7 sequences, the sequences of ST7/allele 137 (MT974101) from strains A1, A3, and A4 were identical to the accession number KF447173 identified in a human from France. One ST7/allele 101 sequence (MT974104) derived from strain N31 and one ST7/allele 99 sequence (MT974105) derived from strain N37 showed 100\% and 99.35\% identity to those in humans in the Czech Republic (MT042799) and Japan (KT438701), respectively. The remaining six ST7 sequences (MT974102, MT974103, and MT974106-MT974109) showed 98.96-99.83\% identity with that from humans in France (KF447173).

\section{Phylogenetic analysis of Blastocystis}

The evolutionary relationship of Blastocystis subtypes found in the present study was analyzed by the neighbor-joining method. The sequences obtained in this study displayed high identity with Blastocystis sequences deposited in GenBank. The phylogenetic tree showed that ST1 was clustered with other ST1 isolated from humans and water samples. ST3 was grouped together with other ST3 identified in humans from different countries. ST7 along with sequences isolated from peafowl and humans clustered together, and formed branches separately (Fig. 2).

\section{Discussion}

The prevalence of Blastocystis in humans varies across countries. Generally, the prevalence in developing countries is higher, ranging from 30 to $100 \%$, compared to developed countries $(0.5 \%-30 \%)[1,26]$. In our study, the prevalence of Blastocystis was relatively low, at $10.1 \%$, compared to other developed countries, such as 33.3\% in diarrheal patients in the United States [27] and 19\% in Australia [28]. However, what is noteworthy is that the prevalence of Blastocystis among the diarrheal samples was much higher as compared to an earlier survey on healthy stool samples from NUH in Singapore (3.3\%) [29]. It is likely that this difference in prevalence is due to the type of sample collected. In this study, only diarrheal samples were collected, since it is the hallmark of CDI, while the criteria for the collection of fecal samples in the earlier study were not as stringent.

On the other hand, we found that among 22 patients who were on concomitant antibiotics, only one was positive for Blastocystis, and of the 25 patients who were positive for Blastocystis, only one was on concomitant antibiotics. There are conflicting reports on age as a risk factor for Blastocystis infection. However, it is noteworthy that the intestinal microbiota changes associated with age [30] and the use of antibiotics may also influence Blastocystis prevalence.

C. difficile is commonly known as a hospital- and antibiotic-associated pathogen and can causes life-threatening diarrhea and colitis [31]. The overall proportion of toxigenic C. difficile $(23.8 \%, 59 / 248)$ from this study was lower than that reported in diarrheal patients from Colombia (57.3\%) [21], but higher than findings in China (14\%) [32] and an early survey in unformed stool samples from Singapore General Hospital (SGH) in Singapore (9.5\%) [33]. Similarly, the proportion of toxigenic C. difficile was 9.6\% (158/1642) from NUH in Singapore [34], and the proportion was $12.5 \%(276 / 2212)$ by both $C$. difficile toxin assay (CDTA) and International Classification of Diseases, Ninth Revision (ICD-9) codes at Tan Tock Seng Hospital in Singapore [35]. Indeed, the prevalence of CDI has increased dramatically worldwide in recent years, especially in Europe and North America [14]. The possible reasons are the widespread use of broad-spectrum antibiotics and the increased prevalence of IBD among others. In addition, the type of test may also affect the prevalence of $C$. difficile. We used enzyme immunoassay to detect the antigen of $C$. difficile, which is an initial test due to its high diagnostic sensitivity, while Vega et al. applied PCR tests with high sensitivity and specificity. This may be one of the reasons for the difference in prevalence found among studies.

Vega et al. reported a significant association between the presence of Blastocystis and CDI in patients with diarrhea [21]. However, we did not find a significant correlation, with only five patients found to be coinfected. Indeed, several clinical studies have indicated that CDI is associated with dysbiosis, and can increase the oxygen content in the intestine [36]. It has been determined that high concentrations of oxygen can affect Blastocystis colonization in the context of dysbiosis [37]. On the other hand, growth of certain facultative anaerobes could also

(See figure on next page.)

Fig. 2 Phylogenetic relationships among nucleotide sequences of Blastocystis partial small subunit ribosomal RNA (SSU rRNA) gene. The neighbor-joining method was used to construct the trees by the Kimura two-parameter model. The number on the branches are percent bootstrapping values from 1000 replicates, with values of more than $50 \%$ shown in the tree. Each sequence is identified by its subtype, host, accession number, and country. Blastocystis subtypes identified in the present study are indicated in bold type 


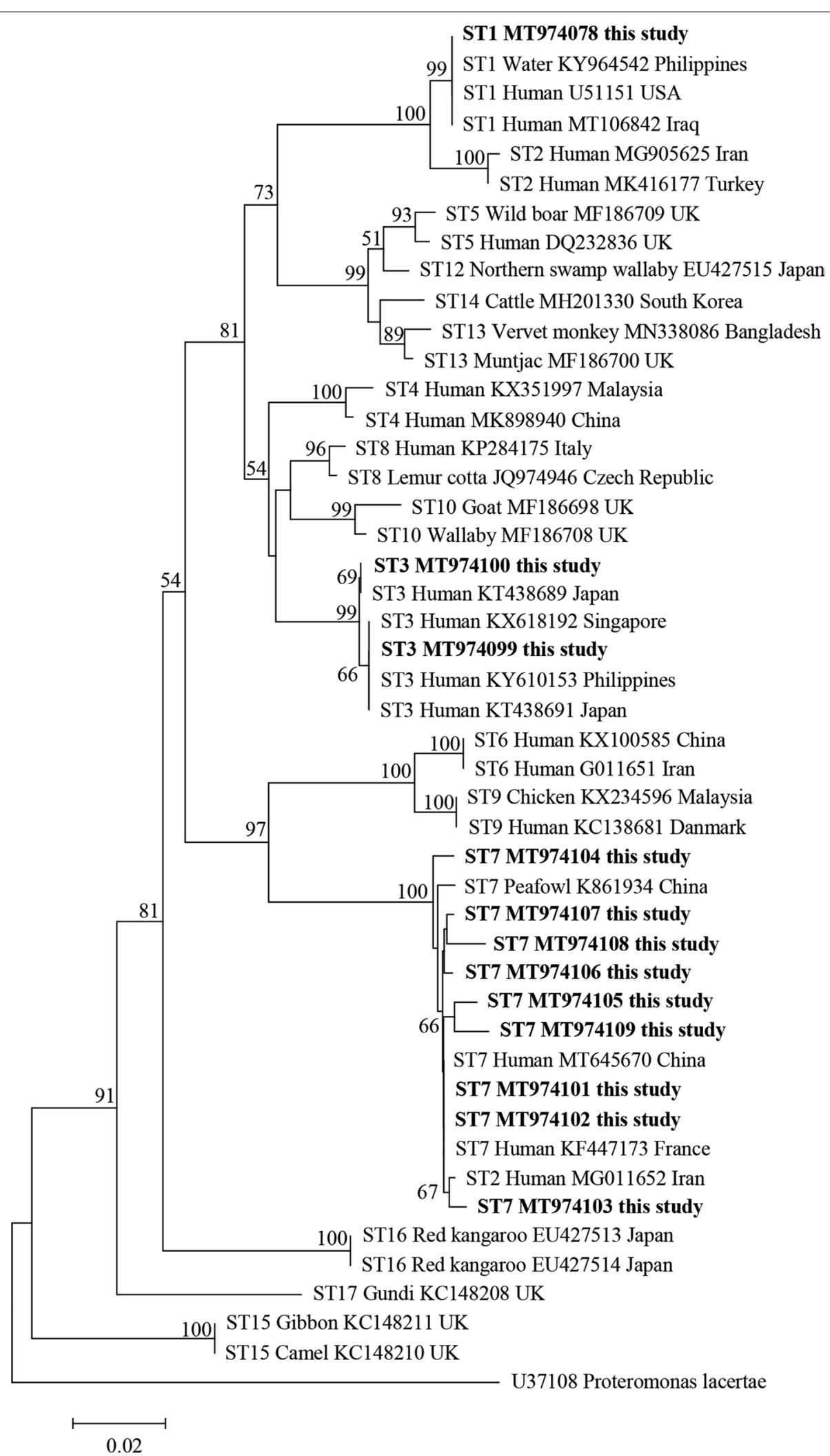


result in high oxygen concentration [38], which may have a detrimental effect on Blastocystis colonization due its anaerobic nature.

Interestingly, the present study revealed that a rare subtype (ST7) was the most prevalent in diarrhea stool samples. A well-studied, pathogenic isolate of ST7 was originally isolated from a patient with gastrointestinal symptoms in Singapore [39], but it has subsequently been identified in humans in many countries, with prevalence ranging from $0.8 \%$ in Nigeria [40] to $17.9 \%$ in Thailand [41]. ST7 has been suggested to be a pathogenic subtype based on in vitro and in vivo mouse studies. It was found to decrease the abundance of beneficial Bifidobacterium and Lactobacillus in a DSS-induced colitis mouse model, leading to a dysbiotic state [12]. Additionally, the cystine proteases produced by ST7 can compromise tight junction proteins zonula occludens-1 (ZO-1) and F-actin in vitro, thereby increasing the permeability of intestinal epithelial cells $[42,43]$. The present study reported high prevalence of ST7 in diarrheal patients, but we were not provided protected health information, and were thus unable to match leukocyte counts and serum creatinine to determine CDI severity, which is a shortcoming of this study.

In this study, considerable intra-ST genetic polymorphisms were found in ST7, and four alleles (allele 99, allele 100, allele 101, and allele 137) were observed in ST7. A more recent study reported ST7 alleles 41, 106, 110, and 112 in gut-healthy humans in the Czech Republic [44]. ST7 was also found in Indian peafowl in China [45], and ST7 (allele 99) was reported in companion animals in a recent study [46], suggesting that ST7 can be transmitted between domestic animals and between animals and humans.

ST1 and ST3 are two common subtypes in humans [47], and are usually associated with healthy gut microbiota. Indeed, these two subtypes are usually identified in asymptomatic patients and can colonize for long periods without any clinical symptoms [48]. Next-generation sequencing (NGS) of the 16S rRNA gene carried out on 2524 subjects in Italy revealed that Blastocystis ST3 carriers were associated with high bacterial diversity and potentially beneficial species such as Prevotella and Ruminococcus [49]. Similarly, ST1- and ST3-colonized individuals are mainly associated with Prevotella and Ruminococcus enterotypes and linked to higher bacterial richness [50]. Notably, Terveer et al. used Blastocystispositive (ST1 and ST3) donor samples to treat recurrent Clostridioides difficile infections (rCDI) through fecal microbiota transplantation (FMT), and demonstrated that the presence of Blastocystis ST1 and ST3 from donors did not cause any adverse gastrointestinal symptoms or have any significant effect on treatment outcome
[51]. Although ST1 and ST3 were also found in this study, their prevalence was very low (3.6\%). More research is needed to better understand the role of Blastocystis ST1 and ST3 in diarrheal patients.

\section{Conclusions}

The present study determined that the prevalence of Blastocystis and the proportion of C. difficile from clinical samples at a local hospital (NUH) were $10.1 \%$ and $43.4 \%$, respectively. Molecular analysis identified three subtypes (ST1, ST3, and ST7), with the geographically rare ST7 as the predominant subtype. Our current study supports the idea that ST7 is a potentially pathogenic subtype, and future research should focus on its relationship with gastrointestinal symptoms and its role in the host intestinal microbiota.

\section{Abbreviations}

C. difficile: Clostridium difficile; PCR: Polymerase chain reaction; ST: Subtype; SSU rRNA: Small subunit ribosomal RNA; IBS: Irritable bowel syndrome; CDI: C. difficile Infection; HAls: Healthcare-associated infections; NUH: National University Hospital; BLAST: Basic Local Alignment Search Tool; ORs: Odds ratios; SGH: Singapore General Hospital; CDTA: C. difficile toxin assay; ICD-9: International Classification of Diseases, Ninth Revision; ZO-1: Zonula occludens-1; rCDI: Recurrent Clostridioides difficile infections; FMT: Fecal microbiota transplantation.

\section{Supplementary Information}

The online version contains supplementary material available at https://doi. org/10.1186/s13071-021-04749-8.

Additional file 1: Table S1. Detailed information about each patient.

Acknowledgements

We would like to thank Tong Jie Xin and Koh Yiling Eileen for critical editing of the manuscript. Lei Deng was the recipient of scholarships from the Chinese Scholarship Council (CSC).

\section{Authors' contributions}

KT conceived and designed the study. Experiments were performed by LD and HT. Fecal samples were collected by JL. Data were analyzed by LD and GP. All authors read and approved the final manuscript.

\section{Funding}

This work was generously funded by MOE Tier-1 grant (R-571-000-037-114).

\section{Availability of data and materials}

The nucleotide sequences generated in present study have been deposited in GenBank (https://www.ncbi.nlm.nih.gov/) under accession numbers MT974098-MT974109.

\section{Declarations}

Ethics approval and consent to participate

This study was approved by the National Healthcare Group Domain Specific Review Board (NHG DSRB) (reference number: 2017/01110). Written informed consent was obtained from the patients before collecting the fecal samples.

Consent for publication

Not applicable. 


\section{Competing interests}

The authors declare that they have no competing interests.

\section{Author details}

${ }^{1}$ Laboratory of Molecular and Cellular Parasitology, Healthy Longevity Translational Research Programme and Department of Microbiology and Immunology, Yong Loo Lin School of Medicine, National University of Singapore, Singapore 117545 , Singapore. ${ }^{2}$ The Key Laboratory of Animal Disease and Human Health of Sichuan Province, College of Veterinary Medicine, Sichuan Agricultural University, 611130, Chengdu, Sichuan, People's Republic of China. ${ }^{3}$ Department of Medicine, Yong Loo Lin School of Medicine, National University of Singapore, Singapore 119228, Singapore. ${ }^{4}$ Department of Gastroenterology and Hepatology, National University Health System, Singapore 119074, Singapore.

Received: 6 January 2021 Accepted: 26 April 2021 Published online: 24 May 2021

\section{References}

1. Clark CG, van der Giezen M, Alfellani MA, Stensvold CR. Recent developments in Blastocystis research. Adv Parasitol. 2013;82:1-32.

2. Tan KSW. New insights on classification, identification, and clinical relevance of Blastocystis spp. Clin Microbiol Rev. 2008;21:639-65.

3. Stensvold CR, Clark CG. Pre-empting Pandora's Box: Blastocystis subtypes revisited. Trends Parasitol. 2020;36:229-32.

4. Stensvold CR, Tan KSW, Clark CG. Blastocystis. Trends Parasitol. 2020:36:315-6.

5. Khaled S, Gantois N, Ly AT, Senghor S, Even G, Dautel E, et al. Prevalence and subtype distribution of Blastocystis sp. in Senegalese school children. Microorganisms. 2020;8:1408.

6. Wu Z, Mirza H, Tan KS. Intra-subtype variation in enteroadhesion accounts for differences in epithelial barrier disruption and is associated with metronidazole resistance in Blastocystis subtype-7. PLoS Negl Trop Dis. 2014;8:e2885.

7. Gentekaki E, Curtis BA, Stairs CW, Klimeš V, Eliáš M, Salas-Leiva DE, et al. Extreme genome diversity in the hyper-prevalent parasitic eukaryote Blastocystis. PLoS Biol. 2017;15:e2003769.

8. Audebert C, Even G, Cian A, Groulap BI, Loywick A, Merlin S, et al. Colonization with the enteric protozoa Blastocystis is associated with increased diversity of human gut bacterial microbiota. Sci Rep. 2016;6:25255.

9. Beghini F, Pasolli E, Truong TD, Putignani L, Caccio SM, Segata N. Largescale comparative metagenomics of Blastocystis, a common member of the human gut microbiome. ISME J. 2017;11:2848-63.

10. Lappan R, Classon C, Kumar S, Singh OP, de Almeida RV, Chakravarty J, et al. Meta-taxonomic analysis of prokaryotic and eukaryotic gut flora in stool samples from visceral leishmaniasis cases and endemic controls in Bihar State India. PLoS Negl Trop Dis. 2019;13:e0007444.

11. Lopetuso LR, Scaldaferri F, Petito V, Gasbarrini A. Commensal Clostridia: leading players in the maintenance of gut homeostasis. Gut Pathog. 2013;5:23.

12. Yason JA, Liang YR, Png CW, Zhang Y, Tan KSW. Interactions between a pathogenic Blastocystis subtype and gut microbiota: in vitro and in vivo studies. Microbiome. 2019;7:30.

13. Céline N, Julien S, Bruno P, Christina NM, Ivan W, Amandine C, et al. Blastocystis is associated with decrease of fecal microbiota protective bacteria: comparative analysis between patients with irritable bowel syndrome and control subjects. PLOS ONE. 2014;9:e111868.

14. Kelly CP, LaMont JT. Clostridium difficile-more difficult than ever. N Engl J Med. 2008;359:1932-40.

15. Bartlett JG, Gerding DN. Clinical recognition and diagnosis of Clostridium difficile infection. Clin Infect Dis. 2008;46(Suppl 1):S12-8.

16. Zimlichman E, Henderson D, Tamir O, Franz C, Song P, Yamin CK, et al. Health care-associated infections: a meta-analysis of costs and financial impact on the US health care system. JAMA Intern Med. 2013;173:2039-46.

17. Shafer CW, Allison JR, Hogue AL, Huntington MK. Infectious disease: health care-associated infections. FP Essent. 2019;476:30-42.

18. Chen X, Lamont JT. Overview of Clostridium difficile infection: implications for China. Gastroenterol Rep. 2013;1:153-8.
19. Hunt JJ, Ballard JD. Variations in virulence and molecular biology among emerging strains of Clostridium difficile. Microbiolo Mole Biol Rev. 2013:77:567-81.

20. Pruitt RN, Chambers MG, Ng KK, Ohi MD, Lacy DB. Structural organization of the functional domains of Clostridium difficile toxins A and B. Proc Natl Acad Sci U S A. 2010;107:13467-72.

21. Vega L, Herrera G, Muñoz M, Patarroyo MA, Ramírez JD. Occurrence of Blastocystis in patients with Clostridioides difficile infection. Pathogens. 2020;9:283.

22. Koh H, Lee MJ, Kim MJ, Shin Jl, Chung KS. Simple diagnostic approach to childhood fecal retention using the Leech score and Bristol stool form scale in medical practice. J Gastroenterol Hepatol. 2010;25:334-8.

23. Quinn CD, Sefers SE, Babiker W, He Y, Alcabasa R, Stratton CW, et al. Diff Quik Chek complete enzyme immunoassay provides a reliable first-line method for detection of Clostridium difficile in stool specimens. J Clin Microbiol. 2010;48:603-5.

24. Scicluna SM, Tawari B, Clark CG. DNA Barcoding of Blastocystis. Protist. 2006;157:77-85.

25. Kumar S, Stecher G, Li M, Knyaz C, Tamura K. MEGA X: Molecular evolutionary genetics analysis across computing platforms. Mol Biol Evol. 2018;35:1547-9.

26. El Safadi D, Gaayeb L, Meloni D, Cian A, Poirier P, Wawrzyniak I, et al. Children of Senegal River Basin show the highest prevalence of Blastocystis sp. ever observed worldwide. BMC Infect Dis. 2014;14:164.

27. Jones MS, Whipps CM, Ganac RD, Hudson NR, Boroom K. Association of Blastocystis subtype 3 and 1 with patients from an Oregon community presenting with chronic gastrointestinal illness. Parasitol Res. 2008;104:341.

28. Roberts T, Barratt J, Harkness J, Ellis J, Stark D, Roberts T, et al. Comparison of microscopy, culture, and conventional polymerase chain reaction for detection of Blastocystis sp. in clinical stool samples. Am J Trop Med Hyg. 2011;84:308-12.

29. Wong KHS, Ng GC, Lin RTP, Yoshikawa H, Taylor MB, Tan KSW. Predominance of subtype 3 among Blastocystis isolates from a major hospital in Singapore. Parasitol Res. 2008;102:663-70.

30. Mariat D, Firmesse $O$, Levenez F, Guimarăes V, Sokol H, Doré J, et al. The Firmicutes/Bacteroidetes ratio of the human microbiota changes with age. BMC Microbiol. 2009;9:123.

31. Abt MC, McKenney PT, Pamer EG. Clostridium difficile colitis: pathogenesis and host defence. Nat Rev Microbiol. 2016;14:609-20.

32. Tang C, Cui L, Xu Y, Xie L, Sun P, Liu C, Xia W, Liu G. The incidence and drug resistance of Clostridium difficile infection in Mainland China: a systematic review and meta-analysis. Sci Rep. 2016;6:37865.

33. Koh TH, Tan AL, Tan ML, Wang G, Song KP. Epidemiology of Clostridium difficile infection in a large teaching hospital in Singapore. Pathol. 2007;39:438-42.

34. Tan XQ, Verrall AJ, Jureen R, Riley TV, Collins DA, Lin RT, et al. The emergence of community-onset Clostridium difficile infection in a tertiary hospital in Singapore: a cause for concern. Int J Antimicrobial Agents. 2014;43:47-51.

35. Chan M, Lim PL, Chow A, Win MK, Barkham TM. Surveillance for Clostridium difficile infection: ICD-9 coding has poor sensitivity compared to laboratory diagnosis in hospital patients Singapore. PLoS One. 2011;6:e15603.

36. Wu Q, Savidge TC. Systems approaches for the clinical diagnosis of Clostridioides difficile infection. Transl Res. 2020;220:57-67.

37. Stensvold CR, van der Giezen M. Associations between gut microbiota and common luminal intestinal parasites. Trends Parasitol. 2018:34:369-77.

38. Rivera-Chávez F, Lopez CA, Bäumler AJ. Oxygen as a driver of gut dysbiosis. Free Radic Biol Med. 2017;105:93-101.

39. Ho LC, Singh M, Suresh G, Ng GC, Yap EH. Axenic culture of Blastocystis hominis in Iscove's modified Dulbecco's medium. Parasitol Res. 1993:79:614-6.

40. Poulsen CS, Efunshile AM, Nelson JA, Stensvold CR. Epidemiological Aspects of Blastocystis Colonization in Children in llero Nigeria. Am J Trop Med Hyg. 2016;95:175-9.

41. Sarinee J, Porntip P, Kookwan S, Somchai P, Arunnee S, Chotechana W, Wachanan W, Hisao Y. Subtype identification of Blastocystis spp isolated from patients in a major hospital in northeastern Thailand. Parasitol Res. 2013;112:1781-6. 
42. Nourrisson C, Wawrzyniak I, Cian A, Livrelli V, Viscogliosi E, Delbac F, Poirier P. On Blastocystis secreted cysteine proteases: a legumain-activated cathepsin B increases paracellular permeability of intestinal Caco-2 cell monolayers. Parasitology. 2016;143:1713-22.

43. Mirza H, Wu Z, Teo JD, Tan KS. Statin pleiotropy prevents rho kinasemediated intestinal epithelial barrier compromise induced by Blastocystis cysteine proteases. Cell Microbiol. 2012;14:1474-84.

44. Lhotská Z, Jirků M, Hložková O, Brožová K, Jirsová D, Stensvold CR, et al. A study on the prevalence and subtype diversity of the intestinal protist Blastocystis sp. in a gut-healthy human population in the Czech Republic. Front Cell Infect Microbiol. 2020;10:544335.

45. Li XD, Zou Y, Pan J, Liang QL, Zeng Z, Meng YM, et al. Prevalence and subtypes of Blastocystis sp. infection in zoo animals in three cities in China. Parasitol Res. 2020;119:465-71.

46. Mohammadpour I, Bozorg-Ghalati F, Gazzonis AL, Manfredi MT, Motazedian MH, Mohammadpour N. First molecular subtyping and phylogeny of Blastocystis sp isolated from domestic and synanthropic animals (dogs, cats and brown rats) in southern Iran. Parasit Vectors. 2020;13:365.

47. Qi M, Wei Z, Zhang Y, Zhang Q, Li J, Zhang L, et al. Genetic diversity of Blastocystis in kindergarten children in southern Xinjiang. China Parasit Vectors. 2020;13:15.
48. Scanlan PD, Stensvold CR, Rajilić-Stojanović M, Heilig HG, De Vos WM, O'Toole PW, et al. The microbial eukaryote Blastocystis is a prevalent and diverse member of the healthy human gut microbiota. FEMS Microbiol Ecol. 2014;90:326-30.

49. Gabrielli S, Furzi F, Fontanelli Sulekova L, Taliani G, Mattiucci S. Occurrence of Blastocystis-subtypes in patients from Italy revealed association of ST3 with a healthy gut microbiota. Parasite Epidemiol Control. 2020;9:e0134.

50. Andersen LOB, Bonde I, Nielsen HB, Stensvold CR. A retrospective metagenomics approach to studying Blastocystis. FEMS Microbiol Ecol. 2015;91:1-9.

51. Terveer EM, van Gool T, Ooijevaar RE, Sanders I, Boeije-Koppenol E, et al. Human transmission of Blastocystis by Fecal Microbiota Transplantation without development of gastrointestinal symptoms in recipients. Clin Infect Dis. 2020;71:2630-6.

\section{Publisher's Note}

Springer Nature remains neutral with regard to jurisdictional claims in published maps and institutional affiliations.
Ready to submit your research? Choose BMC and benefit from:

- fast, convenient online submission

- thorough peer review by experienced researchers in your field

- rapid publication on acceptance

- support for research data, including large and complex data types

- gold Open Access which fosters wider collaboration and increased citations

- maximum visibility for your research: over $100 \mathrm{M}$ website views per year

At BMC, research is always in progress.

Learn more biomedcentral.com/submissions 